\title{
Current Research Situation and Prospect for Thermoplastic Molding Technology of AZ Series Magnesium Alloys
}

\author{
Fang Qian \\ College of Mechanical Engineering, Inner Mongolia University for Nationalities, 028000, China
}

Keywords: Thermoplastic molding technology, AZ series, Magnesium alloys

\begin{abstract}
In this paper, the research status of AZ31 magnesium alloys at home and abroad is reviewed, and the influence of the main alloy elements on the microstructure and properties of AZ31 magnesium alloys is discussed. In this paper, the grain refinement of AZ31 magnesium alloy and the research status of plastic forming technology are introduced, and the development prospect of AZ31 magnesium alloy is analyzed.
\end{abstract}

\section{Introduction}

Magnesium alloy has small density, high specific strength and stiffness, good heat transfer, high conductivity and good electromagnetic shielding and vibration impact absorbing has a series of advantages, etc., are widely used in aerospace, electronics and automobile etc. in different fields, known as green engineering materials in twenty-first Century. At the same time, because of the low melting point, high thermal conductivity and electrical conductivity, active chemical property and easy oxidation and evaporation, magnesium alloys produce a series of welding defects during welding process, which restrict the further development and application of magnesium alloys as structural materials. AZ magnesium alloy has a wide range of applications. Therefore, AZ magnesium alloy is selected as an example. The status and results of research at home and abroad are reviewed, and the effective ways and methods for improving the welding quality of AZ magnesium alloys are discussed. In magnesium alloy materials, most of the finished products are made by casting. For some products with the same structure, other materials can be molded by plastic processing. For $\mathrm{Mg}$ alloy at present level, casting can only be used, because the casting defects can cause the mechanical properties of the castings to be greatly reduced. To apply magnesium alloy more widely, making magnesium alloy components by various deformation methods has become a major trend in the development of magnesium alloys. AZ31 magnesium alloy is the most widely used deformation magnesium alloy at present. $\mathrm{Al}$ is the most commonly used alloying element in magnesium alloys. Strengthening and precipitating phases by solid solution can improve the strength and corrosion resistance of magnesium alloy and reduce the plasticity of the alloy. Mn improves the corrosion resistance of AZ31 magnesium alloys by removing iron and other heavy metal elements to avoid the formation of harmful intercrystallite compounds. In addition, Mn can also refine the grain and improve the weldability. The alloy elements affect the mechanical, chemical and technological properties of magnesium alloys. In most cases, the action of alloying elements is related to the amount of addition. The extent of action is proportional to the amount of added material in the range of solid solubility. When magnesium alloys are used as structural materials, the influence of alloy elements on the processability is much more important than physical properties.

\section{Current Research Situation of Thermoplastic Molding Technology of AZ Series Magnesium Alloys}

Because of the magnesium alloy is close packed six party structure, low symmetry, the axial ratio (c/a) value is 1.623, close to the ideal value of 1.633 close packed, operative slip systems than the FCC and BCC METALS, the magnesium alloy plasticity at room temperature is low, reducing 
forming ability limiting the application of wrought magnesium alloy. Therefore, the research on Super plasticity of magnesium alloys has aroused great attention of scholars from various countries. Magnesium alloys have excellent plasticity and minimal deformation resistance when they are in Superplastic State, which is beneficial to plastic processing. The parts with complex shape or large amount of deformation can be formed at one time, and have the advantages of high fluidity, good filling and small tonnage of equipment. Because there is no elastic deformation in superplastic forming, there will not be spring back after forming, and the precision of workpiece size is high and the surface roughness is small. In a word, superplastic forming is particularly important for materials with high strength and poor plasticity. At present, the research on Super plasticity of magnesium alloys has made great achievements in super plasticity and deformation mechanism, and some superplastic industrial magnesium alloys have entered industrial production.

Mg17Al12 is the only compound phase in the AZ31 magnesium alloy, which is a body centered cubic structure, $a=1.05438 \mathrm{~nm}$. The formation mechanism, the existence state, the number, the size, the distribution of the Mg17Al12 phase and the relationship between the degree and direction of the crystal undoubtedly have an important influence on the properties of the alloys. The research shows that the Mg17Al12 phase can be precipitated from Mg matrix in two ways: continuous and discontinuous precipitation. When the aging temperature is higher than about 205 degrees, the Mg17Al12 phase is precipitated continuously. When the temperature is low, it usually precipitates in discontinuous precipitation. Between Mg17Al12 and matrix orientation relationship were studied in detail. He is taking AZ91 magnesium alloy as base alloy, found by precipitation of solid solution with 4 kinds of morphology and orientation relationship of AZ91 alloy in aging treatment phase, a strip (denoted -Mg17Al12 accounted for more than 90\%), six prismatic, short rod and small equiaxed TEM studies show that the plate strip, six prismatic, short rod shaped precipitates respectively with Burgers, Crawley and magnesium matrix Poter orientation relationship between particle phase between particles with precipitation or precipitation can be formed with dissimilar twin structure, pseudo twin relationship. The precipitation of precipitates in the vertical direction will effectively improve the strength of the alloy. At present, the researchers have done a lot of work in improving the microstructure and improving the performance of AZ (Mg-A1-Zn) alloy. Due to the low content of alloy elements in AZ31 deformation, the effect of solid solution strengthening and aging strengthening is not obvious. Therefore, the purpose of research is to improve the microstructure by means of alloying of trace elements and composite alloying. Trace elements can further improve the properties of Mg-A1-Zn alloy at room temperature and high temperature by improving the morphology and structural characteristics of alloy phase, forming new secondary phase with high melting point and high thermal stability, or refining grain structure. Many studies have shown that Ca, $\mathrm{Sr}, \mathrm{Sn}, \mathrm{Be}, \mathrm{Sb}, \mathrm{Y}, \mathrm{Nd}$, Ce and other trace elements can effectively improve the microstructure of the AZ - based magnesium alloy.

Studies have shown that when the Ce content is less than 1\%, AZ31 magnesium alloy with the increase of Ce content, the eutectic in the magnesium matrix on the grain boundary is also increasing, when the content reaches $1 \%$, the eutectic phase in the magnesium matrix grain gradually into the mesh prevents the grain growth, grain refinement, tensile strength and plastic has been significantly improved. Wang Lingyun et al. in Chongqing University, adding Ca and Sr into AZ31 wrought magnesium alloy, the casting grain structure of 40 50 m was obtained, and the subsequent process ability and mechanical properties of the alloy were obviously improved. There is a functional relationship between the yield strength and the grain size of the material. The $\mathrm{K}$ value increases with the increase of the Taylor coefficient. Because magnesium is a closely packed six party structure, compared with the face centered cubic and the body centered cubic structure, the Taylor coefficient of magnesium is large, and the $\mathrm{K}$ value is also large. Therefore, the grain refinement of magnesium alloys is an important technical measure to improve the mechanical properties of magnesium alloys. The grain refinement can be divided into two aspects, on the one hand, the grain refinement of the cast state and the refinement of the morphed grain.

The research on the refinement method of as cast grain mainly concentrates on choosing suitable grain refiners and adding methods. For AZ series alloys, there are many grain refiners, such as $\mathrm{C}, \mathrm{Ca}$ 
and Sr. The existing state and adding mode of the refiner have a significant effect on the refining effect. Zhang Shijun put forward the new way for magnesium alloy powder grain refiner was added son mother ball lump of mixed powder addition method, it is aluminum powder and carbon powder according to a certain proportion of flux and mechanical mixing together, with aluminum foil into balls, and then press the ball wrapped with 600MPa pressure press magnesium alloy molten by bell, to be completely melting after pouring toner, the toner adding method can improve the refining effect on magnesium and its alloys. For the variation of grain refinement, Chongqing University Wang Lingyun from the control of dynamic recrystallization grain size of AZ31 magnesium alloy at 300 450 DEG $C$ and the strain rate of 0.1 and 1.0s-1 under the condition of hot compression, found that dynamic recrystallization occurred during hot compression deformation, the dynamic recrystallization (average grain size the natural logarithm of D) and Zener-Hollomon parameters (z) natural logarithm.

\section{Prospect for Thermoplastic Molding Technology of AZ Series Magnesium Alloys}

Magnesium alloys are widely used as structural parts, and their performance requirements are constantly improving. Guided by the continuous development of magnesium alloys, the research of magnesium alloy welding will still be one of the key areas to expand the application area of magnesium alloys. The electron beam welding, TIG welding, friction stir welding and laser -TIG composite welding technology can get the weld joint with beautiful appearance and better joint performance. However, the generation of brittle and hard phase and the existence of weld residual stress are all harmful to the joint performance. During the welding process, the occurrence of brittle and hard phases in the weld area and the elimination of residual stress after welding or the occurrence of residual stress in welding process need to be solved. The weld zone grain coarsening, porosity formation and a series of welding problems is also the focus of research, at the same time, the main content development method of welding of magnesium alloy and magnesium alloy connecting with other metals and the principle of research is to further enhance, application of magnesium alloy material. There is less research on the welding of magnesium alloys in the middle layer, and it will be an important research content to carry out the study of composite welding process with intermediate layer. AZ31 magnesium alloy has many unique advantages, has been widely used in aerospace, automotive, 3C and other fields. But some disadvantages of it also limits the application of magnesium alloy AZ31, making it the potential is far from fully. The author believes that future work should start from the following aspects: basic research on AZ31 magnesium alloy. Including grain refinement of alloy, alloy based on the existing research on improved forming technology of.AZ31 magnesium alloy. The deformation mechanism of the superplastic. Especially the research on the develop process and reasonable processing parameters. The strain rate superplastic forming technology has the important significance of.AZ31 magnesium alloy research on composites of high stress. Although the magnesium based composite material has made some achievements, but on the application of magnesium alloy to enhance the research and development of body and its interface theory still exist the limitation, especially the low strength of magnesium matrix composites, has become a major obstacle to development. If the mechanical properties of matrix materials can be improved, and then combined with composite strengthening means, we hope to develop high strength magnesium matrix composites.

Strict control of smelting and casting process to obtain high quality ingot is the basis of ensuring the smooth plastic forming of magnesium alloy. For qualified ingot, a series of pressure processing means, such as extrusion, forging and rolling, can be used for subsequent deformation. These pressure processing methods are like those of aluminum alloys, but the most basic difference between magnesium and magnesium alloys and other metal materials in plastic forming technology is that deformation needs to be carried out at a certain temperature. AZ31 has good deformation ability and can adopt a variety of deformation methods. 1, extrusion technology has been continuously improved and improved in recent years. Besides the traditional positive and negative extrusion, many new technologies and methods have been developed to enhance extrusion process, such as cold extrusion, 
perforation extrusion, lubrication and extrusion. At present, the research on AZ31 magnesium alloy isothermal extrusion as compared. The results show that the grain of AZ31 magnesium alloy is obviously refined by extrusion deformation, and its strength, hardness and elongation are greatly improved. Magnesium alloy is brittle fracture when temperature is below 200. It is easy to occur corrosion and oxidation and grain coarsening at above 400. Magnesium alloy has large thermal conductivity and narrow forging temperature range. Magnesium alloy sheet is usually produced by rolling method. Temperature is the most important process parameter in the rolling process of magnesium alloy. During low temperature rolling, high stress concentration can lead to twin nucleation and shear fracture.

When magnesium alloy is rolled, the temperature range is generally 225 450 degrees C. Under the temperature range and the medium strain rate, dynamic recrystallization is easy to occur, but the rolling properties are obviously improved. There are many kinds of rolling methods for 31 Magnesium Alloys, such as asynchronous rolling of equal diameter angle rolling, etc. All the rolling methods can refine the grain of magnesium alloy, make the microstructure uniform and improve the mechanical properties. Because the technology of magnesium alloy is closely arranged six party structure superplastic forming, low symmetry, the axial ratio (c/a) value is L623, close to the ideal value of close packed L633 operative slip systems than the FCC and BCC METALS, the magnesium alloy plasticity at room temperature is low, reduced forming ability, limiting the application of wrought magnesium alloy. AZ31 magnesium alloy is mainly formed by controlling deformation such as rolling, extrusion or forging, and obtains mechanical properties such as strength and plasticity required by controlling heat treatment state. The mechanical properties of AZ31 magnesium alloy plate, extrusion bar and profile, and the typical room temperature mechanical properties of AZ31B magnesium alloy thick plate and thin plate heat treatment state. The mechanical properties of AZ31 magnesium alloy at room temperature are relatively low, which greatly limits its popularization and application.

It is urgent to improve the mechanical properties of the alloy. The mechanical properties of magnesium alloy can be effectively improved by suitable processing technology. The plastic deformation behavior of magnesium alloy AZ31 magnesium alloy AZ31 alloy slab was rolled to study its plastic deformation by extrusion making magnesium alloy AZ31 magnesium alloy slab. The welding effect of the composite heat source welding magnesium alloy weld is increased significantly, which is 2 times the penetration depth of the TIG welding alone under the same welding specification. The composite arc stability is better, the maximum speed of the composite heat source current steady combustion is increased by more than 1 times than the single arc. The maximum shear strength of 193.3MPa laser -TIG hybrid welding seam welding of magnesium alloy joints, the tensile strength of the base metal is about $80.1 \%$. with a laser beam as heat source welding of magnesium alloy, the weld is easy to produce pores, evaporation and oxidation, the welding deformation and stress concentration. Therefore, we should improve laser welding technology, improve the quality of welding equipment, control the nucleation and growth of welded joints, improve the stress concentration and improve the joint performance. Magnesium alloys have many excellent properties, as the structure of the broad application prospect of magnesium alloy, the welding characteristics become the bottleneck as the structure of deep processing of magnesium alloy welding in recent years more and more research, research on the welding of AZ31B magnesium alloy have also done a lot of work, the main method of welding brazing, electron beam welding TIG welding, laser welding, friction stir welding, diffusion welding and welding methods and effect of composite welding etc.. The connection of AZ31B magnesium alloy welding method. The characteristics and give full play to their respective welding methods, to improve the study on the interaction mechanism of joint performance. It is further improved the theoretical basis of hybrid welding and welding process of special flux and development, which is also one of the hot research in the future. 


\section{References}

[1] Chen Xianhua, Xiao Rui, Ding Xuezheng, et al. Research on AZ Alloy [J] Hot Working Technology, 2012, 41(4): 14-18.

[2] Lin Weili. Research Status and Outlook of High Strength and Ductility Mg-RE Magnesium Alloys [J] Hot Working Technology, 2015, 44(4): 12-24.

[3] Xiao Pan, Liu Tianmo, Jiang Dan.Research Status and Development of AZ31 Magnesium Alloy [J] Journal of Chongqing University (Natural Science Edition), 2006, 29(11): 81-84.

[4] Liu Fenjun, Wang Hanying. Research status and development tendency of welding technology of AZ31B magnesium alloy [J]Ordnance Material Science and EngineerinG, 2013, 36(5): 129-132. 